\title{
A Novel Method for Very-high Frequency Omni Range (VOR) Azimuth Error Correction using Kalman Filter for the Improvement of Aircraft Density
}

\author{
K. Ramakrishna ${ }^{1}$, M R K Murthy ${ }^{2}$, N S Murthy Sharma ${ }^{3}$ \\ Research Scholar, Dept of ECE, College of Engg., Osmania University, Hyderabad, India ${ }^{1}$ \\ Dept of ECE, College of Engg., Osmania University, Hyderabad, India ${ }^{2}$ \\ Dept of ECE, SNIST, Ghatkesar, Hyderabad, India ${ }^{3}$
}

\begin{abstract}
A novel method for error correction for Very-high frequency Omni Range (VOR) Azimuth is designed and tested. The basic principle behind the working of VOR is enumerated. In the generation of phase difference at the airborne receiver, various errors occur which impair the effective working of VOR. The aggregate value of these errors will be around $\pm 5^{\circ}$. Broadcom BCM2836R processor is used in the Linux environment with Mathematica10. Wolfram language is used to realize Kalman filter. Incorporating the proposed method, the aggregate error is successfully reduced to around $\pm 1^{\circ}$. This improvement will benefit the air operators to have more aircraft density surrounding any airport/VOR area.
\end{abstract}

Keywords: Kalman filter, Very-high frequency Omni Range (VOR), Azimuth, Wolfram language.

\section{INTRODUCTION}

This paper discusses various aspects regarding VOR function, its principles, wave pattern including functional description of VOR in general and various errors involved in the functioning of the VOR in particular are touched upon with a specific eye on improving the errors involved in the functioning of the VOR, especially after analysing the errors in ground check, course, station errors, side band errors, antenna errors, deruntal/ quadrantal errors and propogational effect errors.

VOR is the most significant aviation invention in 1950s[1]. By using VOR, pilot can accurately navigate from point A to point B. There are two types of VOR, namely conventional VOR (CVOR) and Doppler VOR (DVOR). Even though both serve the same purpose as far as the aircraft are concerned, the selection of the CVOR and DVOR depends upon various site conditions in an airfield. The basic concepts of CVOR will be discussed. Here VOR theory, significance of VOR, generation of space wave pattern on the radiated signal is presented. The detailed error analysis of VOR is carried and the possible solutions to reduce the errors using Kalman Filter are offered.

A. Omni-Range

Omni-range is based on the radiation of two signals. One of the signal is transmitted Omni directionally modulated with carrier is known as reference signal. The second transmitted signal would have a field pattern with the shape of the figure of eight (8) but is not modulated. The Omni pattern is continuously rotated. If the pattern is rotated very slowly, an observer at a fixed point could record the values of the field strength on a meter. He would record exactly the same relative readings as if the pattern is fixed in the space and as he walks around the antenna with the indicating equipment. In one revolution of the figure-of-eight pattern the indicating meter would pass through two maxima and two null points in a sinusoidal manner. If this rotation is accomplished at a speed corresponding to the frequency of the reference signal modulation, the output of the indicator would be an audio note with the frequency equal to the reference signal modulation frequency. If the equipment is adjusted such that the voltage of the modulation frequency of the reference signal is a maximum as the figure of eight is passing through the north, then the audio frequency and the output frequency from the indicator will always be in phase, when observer is standing at north of the radio station. If now observer move towards east, his indicator will be at zero voltage when the modulating signal voltage is maximum therefore by reading the phase existing between the reference signal and variable signal the bearing of the observer's position with respect to the radio station can be determined directly [1].

A system of this type could be made to operate by transmitting the reference and variable phase signal on two different carrier frequencies that are received on separate receivers. To avoid the generation of two carriers, only one carrier 


\section{International Journal of Innovative Research in Electrical, Electronics, Instrumentation and Control Engineering \\ ISO 3297:2007 Certified}

Vol. 5, Issue 6, June 2017

frequency is used and other carrier modulated by $10 \mathrm{KHz}$ is known as sub carrier. This means that at a spacing of $10 \mathrm{KHz}$ from the carrier that appears as a separated signal which can be received over the same radio receiver. This sub carrier is frequency modulated by a $30 \mathrm{~Hz}$ signal and its receiption and demodulation produces the reference signal. An un modulated carrier is applied to a goniometer rotating at $1800 \mathrm{rpm}$ which is connected to four vertical antennas to produce the variable phase signal.

The application of Kalman filter is not so far realised for the VOR scenario. Hence, the incorporation of the algorithm to this context is claimed as novel.

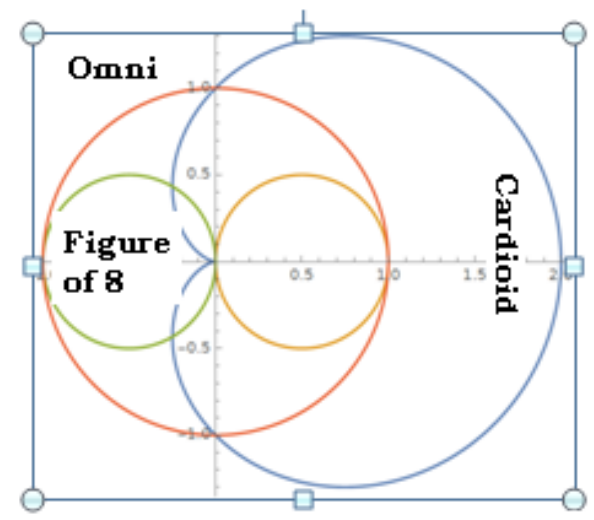

Figure: 1 Generation of a cardioids

\section{GENERATION OF SPACE WAVE PATTERN}

To generate the required wave shape for Omni range i.e. cardioid field pattern, two types of wave pattern are required. They are Omni and figure of eight pattern. The figure of eight has two maxima and two minima positions in $360^{\circ}$ rotation. This dual indication is undesirable. A field pattern having the form of cardioid would be more desirable because there would be one maxima and one minima. The mathematical expression of figure-of-eight is

$$
\mathrm{E}=\mathrm{E}_{\max } \cos \theta
$$

Where $\mathrm{E}$ is conventional Electric field intensity in $\mathrm{V} / \mathrm{m}$. However its designated usually as applied voltage output of the loop having a maximum value of $\mathrm{E}_{\max }$ in aircraft operating books. when the plane of the loop in the line with the direction from the centre of the loop to the source of the radiant energy. A simple vertical antenna has equal output in the horizontal plane for any angle between a plane containing the vertical antenna and the direction of the radiation of the energy source. Its field pattern is circle and the equation can be presented as

$$
\mathrm{E}=\mathrm{E}_{\max }
$$

Adding the above equations, we get

$$
2 \mathrm{E}=\mathrm{E}_{\max }(1+\cos \theta)
$$

The above equation (3) will generate the cardioid pattern which is the required Omni range space pattern. If we vary the value of $\theta$ from 0 to $2 \pi$, it can be seen that there is only one angle for which the output is maximum and only one for which the output is zero. This is represented in Fig.1, along with figure of eight and Omni field patterns

\section{VHF OMNI RANGE PRINCIPLES}

Radiation from the Omni range system antenna consists of two VHF carriers that provide navigation, identification and speech information as shown in Fig.2. The navigational portion requires the combined use of two separate carriers, a reference carrier and a variable carrier, each originates from a common transmitter. Although the two carriers are on the same VHF frequency, they differ in that the reference carrier contains a sub carrier modulation and is radiated in a nondirectional manner about the antennas. The variable carrier contains no modulation but is radiated from the antenna in the form of a revolving eccentric lobe that rotates at 1800rpm. Thus a receiver placed at a given point of azimuth receives a signal which increases and decreases in amplitude at a $30 \mathrm{cps}$ rate. The amplitude variations occur sinusoidally and produce an audio signal which is very nearly a pure sine wave at the demodulated receiver output. The keyed tone station identification and speech signals are applied to the reference carrier is AM modulation in a conventional manner (see Fig.3)[4]. 


\section{International Journal of Innovative Research in Electrical, Electronics, Instrumentation and Control Engineering \\ ISO 3297:2007 Certified}

Vol. 5, Issue 6, June 2017

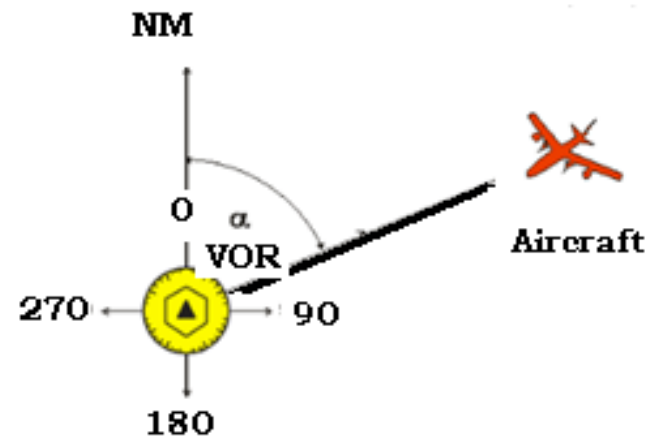

Figure:2 Principle of operation of VOR

Phase comparison omni range.

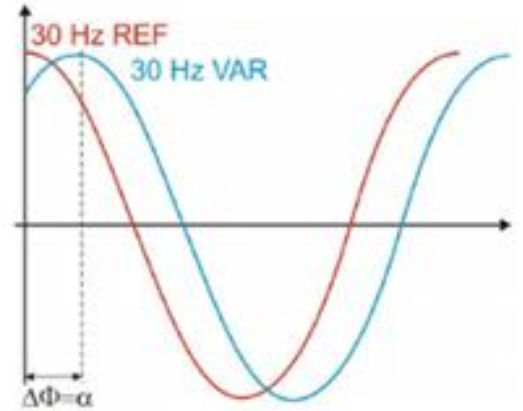

Figure:3 Detected $30 \mathrm{~Hz}$ FM by Aircraft recerver approaching VUK

In the field when the aircraft is at the angle $\alpha$, with respect to the NM, the same is reflected as the phase difference between the Reference and the Variable $30 \mathrm{~Hz}$ signals, in the aircraft.Omni range operation is based on phase comparison at the aircraft receiver and as such requires that the Omni range station provide two signals which have a correlation between phase and degree of azimuth around the station site, the degrees of azimuth to be synchronized with magnetic north. Because of the rotational characteristics of the variable carrier, the $30 \mathrm{~Hz}$ variable phase receiver output has azimuth sensitivity, which is measurable. The reference carrier provides a magnetic north (zero degree) reference phase by virtue of the sub carrier modulation applied. This sub carrier modulation consists of a $9960 \mathrm{~Hz}$ signal FM modulated plus and minus $480 \mathrm{~Hz}$ at a $30 \mathrm{~Hz}$ rate. The sub carrier signal is applied to the reference carrier as AM modulation, and the $30 \mathrm{cps}$ reference phase output of the receiver demodulator is in -phase with the 30cps variable phase at magnetic north only [4].

Receiver placement at specific points of azimuth around the Omni range antenna results in a varying amount of lag of the $30 \mathrm{cps}$ variable phase behind the reference phase. This lag is due to the time element required for the variable carrier to be to rotate around the azimuth to the receiver location. At a point 90 degrees from the receiver location the variable phase signal is at zero amplitude, as the lobe rotates towards the receiver location, and passes through maximum. Further rotation of the lobe causes the variable phase output to go in the negative direction, through maximum to zero, to complete the cycle. The conditions just described are present due to 'space modulation', which is the algebraic addition that takes place between the reference \& variable carriers.

\section{A. Station identification and Voice}

The 1020-cycle code identification signal or the voice signals amplitude modulates the transmitter output in the same manner as the 9960-cps reference modulation. These signals appear on the circular radiation pattern and will be received with equal intensity in any direction from the station. Tone identification is adjusted to use 10 percent of the modulation capacity, and voice signals are adjusted to use 30 percent of the modulation capacity. When voice modulation is used, the tone identification is removed by the circuitry activated by operation of the microphone pressto-talk bar and reinserted when the press-to-talk bar is released.

B. Navigational Information Signal Path

A 9960 reference sub carrier is generated in the goniometer and is frequency modulated at a $30 \mathrm{~Hz}$ rate by a rotating capacitor. The reference sub carrier is applied to the transmitter modulator, via a fixed attenuator-mixer pad in the local 


\section{UGC Approved Journal}

IJIREEICE

\section{International Journal of Innovative Research in Electrical, Electronics, Instrumentation and Control Engineering \\ ISO 3297:2007 Certified}

Vol. 5, Issue 6, June 2017

control unit, and amplitude modulates the VHF carrier developed in the transmitter. This modulated carrier passes through a coaxial relay to the reference section of the antenna and is radiated Omni-directionally.

A portion of the transmitter output is fed to the modulation eliminator, where the modulation is removed. Output of the modulation eliminator passes through the goniometer VHF capacitor which divides the signal into two equal parts displaced 90 degrees in phase and varying sinusoidally in amplitude at a $30 \mathrm{~Hz}$ rate. Output of the goniometer capacitor is connected through separate coaxial relays to the two variable section of the antenna and radiated in the form of a rotating figure of eight pattern. The combined radiation (total of reference and variable carrier radiations) produces a rotating pattern, which carries distinctive bearing information along any radial (magnetic bearing) from the Omni range station.

\section{VOR ERROR SIGNAL CONCEPT}

This section presents a brief review of the conventional VOR (CVOR) and signal reflection error which impair the effectiveness of the VOR under adverse sitting conditions.

The conventional VOR provides navigational signals for an aircraft receiver which will allow the pilot to determine the bearing of his aircraft relative to a VOR facility. The bearing information is called the Omni course and is determined by measuring the phase difference between two detected $30 \mathrm{~Hz}$ signals in the aircraft.

The constant phase $30 \mathrm{~Hz}$ reference signal is transmitted as frequency modulation of a $9960 \mathrm{~Hz}$ sub carrier (often referred to as a $10-\mathrm{KHz}$ sub carrier) which, in turn, amplitude modulates the VOR transmitter and is radiated Omni directionally from the VOR. In the aircraft receiver, the $9960 \mathrm{~Hz}$ FM sub carrier is amplitude detected from the VOR carrier. The FM sub carrier is then frequency detected to produce the $30 \mathrm{~Hz}$ reference bearing signal. The detected $30-$ cpc reference signal has a constant phase at all azimuths about the VOR.

The 30-cps variable signal is produced by space modulation of the radiated RF carrier energy. When detected in the aircraft receiver, the 30-cps signal has a variable phase around the VOR, its phase changing one degree for each degree change in azimuth.

An errorless VOR system is designed in such a manner that the reference and variable 30-cps signals are received in phase at a zero degree or north bearing from the VOR. As the receiving point is moved clockwise around the VOR, the detected variable 30 -cps signal begins to lag the detected reference 30-cps signal. If there are no system errors, the variable signal will lag the reference signal one degree for every one degree of clockwise movement around the VOR. The receiver will give an on-course indication when the two 30-cps signals are put back in phase by receiver circuitry. This is done when the Omni bearing selector control is adjusted in the receiver. The course read on the receiver dial indicates the amount of phase shift required to put the two 30-cps signals in phase. The phase relationship of the two navigation signals is independent of the heading and altitude of the aircraft and, therefore the variable signal shift in phase according to the bearing of the aircraft relative to the VOR.

\section{VOR ERROR ANALYSIS}

There are six causes for an error in the VOR reading. Three because of instrument errors and the other three are because of propagation errors. The three instrument errors are :

receiver indication error, polarisation error, and ground station error. The three propagation errors are vertical pattern error, terrain effect, and site effect.

Instrument errors:

1. Receiver errors are due to failure of receiver equipment to translate the bearing information accurately. This error is mainly because of unequal phase shifts in receiver channels for variable phase and reference phase signal errors in the phase detector, or because of inaccuracy in resolver.

Receiver indication errors are determined by bench calibration. Any discrepancy in calibration reflects in indication errors. The receiver error exhibits, slow and systematic variation with bearing, in the best receivers these errors vary between 0.5 to 1.0 degrees.

2. The presence of appreciable vertical component is the main cause of polarisation error. This is often called the 'pushing effect' or 'altitude effect'. Error due to vertical field is given by $\theta=\tan ^{-1}\left(\frac{E_{v}}{E_{h}}\right)$

In the above equation $\theta$ is the error in degrees. $E_{v}$ is the vertically polarised field strength and $E_{h}$ is the horizontally polarised field strength. This error will be approximately $1.5^{\circ}$ to $2.6^{\circ}$. To reduce this error the vertical field should be suppressed both at transmitting and receiving antennas by at least $20 \mathrm{~dB}$. 


\section{International Journal of Innovative Research in Electrical, Electronics, Instrumentation and Control Engineering \\ ISO 3297:2007 Certified}

Vol. 5, Issue 6, June 2017

3. Ground station errors are because of unbalanced side band powers, antenna phase, improper earthling or of counterpoise and misalignment of North Mark. The distance between correct position and indicated bearing is known as error. Forward and reverse ground checks can be carried out to separate gonio error from antenna error.

In order to get explanation for derivation of various errors and probable causes, station goniometers and antenna error curves can be studied. However course error spread for each VOR transmitting equipment shall not exceed $\pm 3^{\circ}$.

VOR antenna errors are caused because of, incorrect antenna tuning, defective antenna coaxial feed line, and antenna sideband feed lines out of phase with each other and also out of phase with reference to feed line and defective corroded parts and connections on interior of antenna.

Propagation effect errors: The vertical pattern effect is very significant, whenever the reflections are prominent in a VOR site and can be reduced if we elevate the VOR hut to a maximum relative height so that the reflected signals can be reduced.

In the terrain errors it is found that the path of the propagation is non uniform and also the refractive index of the medium change can be abrupt as the change over from ground to sea causes path distortion. Site effects are also a major cause for VOR signals which are to be minimized for better performance of VOR.

The algebraic sum of all the errors discussed above is termed as aggregate error. . Precise estimation of each error is an extremely difficult task, even on probability basis. But the main purpose of the analysis and estimating these errors is to bring out the point that VOR is not a precision navigational-aid. It is found in practice that all the errors put together, the total error of more than $\pm 5^{\circ}$ is seldom encountered. The values of aggregate error and the pilot error are put together in the determination of standards used for aircraft separation purposes.

From the above VOR error analysis we take the maximum error of $\pm 5^{\circ}$ is the maximum aggregate error and we have reduced this error using Kalman filter algorithm so that the VOR nav-aid facility can be used for better separation of aircraft while vectoring.

\section{DATA SIMULATION}

VOR data is simulated using Mathematica10.built in function "RandomInteger" [5][6]. We have taken 12 azimuth steps of $30^{\circ}$ each starting from $0^{\circ}$ to $360^{\circ}$.(see Tab.1).

Table 1 : VOR Data Simulation( deg)for $0^{\circ}$ to $360^{\circ}$ from MM

\begin{tabular}{|l|l|l|l|}
\hline $\begin{array}{l}\text { Actual } \\
\text { Bearing }\end{array}$ & $\begin{array}{l}\text { Simulated } \\
\text { Bearing }\end{array}$ & $\begin{array}{l}\text { Corrected } \\
\text { reading }\end{array}$ & Error \\
\hline 0 & -7 & -7 & 7 \\
\hline 30.00 & 21 & -9 & 9 \\
\hline 60.00 & 70 & 10 & 10 \\
\hline 90.00 & 87 & -3 & 3 \\
\hline 120.00 & 130 & 10 & 10 \\
\hline 150.00 & 160 & 10 & 10 \\
\hline 180.00 & 181 & 1 & 1 \\
\hline 210.00 & 209 & -1 & 1 \\
\hline 240.00 & 231 & -9 & 9 \\
\hline 270.00 & 276 & 6 & 6 \\
\hline 300.00 & 294 & -6 & 6 \\
\hline 330.00 & 324 & -6 & 6 \\
\hline
\end{tabular}

At each step of the azimuth, we have generated $\mathrm{a} \pm 5^{\circ}$ random number on either side of the azimuth, to simulate the VOR signal from $0^{\circ}$ to $360^{\circ}$.

This signal is applied as a measured value Z[n], in Kalman Filter algorithm

Using Wolfram Language, program is written for Kalman Filter for the following:

1. $\mathrm{K}[\mathrm{n}]$, for Kalman gain taking error in measurement and error in estimation as input parameters.

2. $\mathrm{X}[\mathrm{n}]$, for expected value taking previous expected value $\mathrm{X}[\mathrm{n}-1]$, and current measured value $Z[n]$, as inputs.

3. $P[n]$, for error in expected value, taking current Kalman gain K[n], and previous error in expected value $P[n-1]$, as inputs.

The above values are tabulated for 12 iterations for record, analysis and discussion 
UGC Approved Journal

IJIREEICE

\section{International Journal of Innovative Research in Electrical, Electronics, Instrumentation and Control Engineering \\ ISO 3297:2007 Certified}

Vol. 5, Issue 6, June 2017

\section{RESULTS, ANALYSIS AND DISCUSSION}

The above simulated values are used as measured values in the Kalman filter algorithm to get the gain (K), expected value $(\mathrm{X})$ and error in expected value $(\mathrm{P})$ for each iteration which is tabulated in the below Table.2. Form the below table it can be seen that the error in expected value is gradually decreased in an exponential manner. Perusal of the table shows that the error in the expected value is getting reduced for each iteration from 9.47 to 1.5 Hence we can say that application of Kalman Filter for VOR estimates will clearly reduce the azimuth error. We have also drawn a comparative picture of True bearing, Measured value and the Expected value in the following Fig.4.

Table 2 : Kalman Filter output with Kalman gain, Expected value and Error in Expected

\begin{tabular}{|l|l|l|}
\hline $\begin{array}{l}\text { k } \\
\text { Kalman } \\
\text { Gain) }\end{array}$ & $\begin{array}{l}\mathbf{x} \text { Value (Draw) } \\
\text { (Expected } \\
\text { Value) }\end{array}$ & $\begin{array}{l}\text { p Error } \\
\text { in } \\
\text { Expected } \\
\text { Value) }\end{array}$ \\
\hline 0.474 & 15.3 & 9.47 \\
\hline 0.321 & 19.2 & 6.4 \\
\hline 0.243 & 17.5 & 4.9 \\
\hline 0.196 & 38.6 & 3.9 \\
\hline 0.164 & 52.0 & 3.3 \\
\hline 0.141 & 68.0 & 2.8 \\
\hline 0.123 & 80.0 & 2.5 \\
\hline 0.110 & 98.0 & 2.2 \\
\hline 0.099 & 108.0 & 2.0 \\
\hline 0.090 & 118.0 & 1.8 \\
\hline 0.083 & 133.0 & 1.7 \\
\hline 0.076 & 160.0 & 1.5 \\
\hline
\end{tabular}

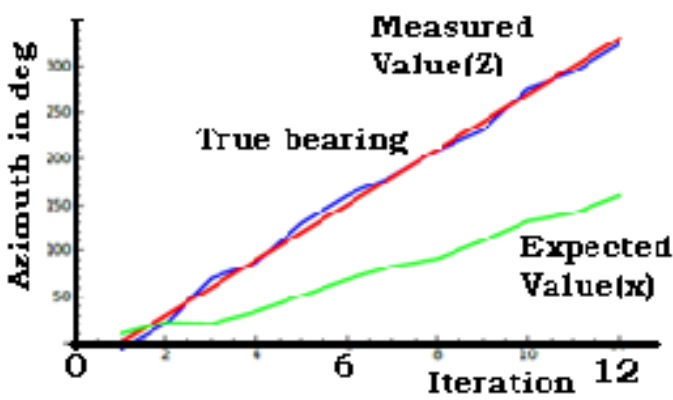

Figure 4: Kalman Filter output along with true and measured azimuth of VOR from $0^{\circ}$ to $360^{\circ}$ in 12 iterations

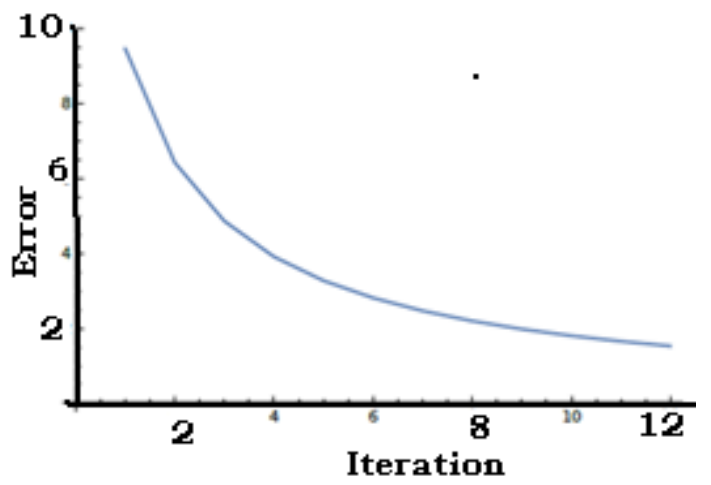

Figure 5: Error in expected value for 12 iterations 


\section{International Journal of Innovative Research in Electrical, Electronics, Instrumentation and Control Engineering ISO 3297:2007 Certified}

Vol. 5, Issue 6, June 2017

The error in expected value for the twelve iterations is drawn in the Fig.5. From the Fig.5 we can easily see that, the azimuth error of VOR is reduced from 10 units to 2 units. In other words the error reduced from $\pm 5^{\circ}$ to $\pm 1^{\circ}$. This is a very significant figure, so that the number of aircraft in one square mile (the aircraft density), can be increased significantly. Consequently the flight time and the cost of operations can be reduced.

The above results are obtained in LINUX environment, with Wolfram Mathematica 10 and on a Broadcom 2836 system, with a $900 \mathrm{MHz}$ crystal.

The Kalman Filter was observed to be slow after 10 iterations.

This problem can be solved if we use a 2 or $3 \mathrm{GHz}$ system, so that the entire setup can be used in real-time.

\section{CONCLUSIONS}

The application of Kalman filter is not so far realised for the VOR scenario. Hence, the incorporation of the algorithm to this context is claimed as novel. A unique method for error correction for Very-high frequency Omni Range (VOR) Azimuth is designed and tested successfully. The applications of VOR as both enroute short distance aid as well as holding point at International Airports are discussed with a particular reference to CVOR. In the generation of phase difference at the air-borne receiver, instrument and propagation errors occur which impair the working of VOR. The aggregate value of these errors is found to be around $\pm 5^{\circ}$. A Kalman Filter Algorithm was devised and executed to reduce the aggregate error to $\pm 1^{\circ}$. This improvement will benefit the air operators to have more aircraft density surrounding any airport/VOR area. In turn this will economise the operations. Broadcom BCM2836R processor used in the Linux environment with Mathematica10. and Wolfram language.

\section{Appendix}

A. 1 Radio navigational aids

The navigational aids are commonly known as nav-aids which are a set of ground based facilities provide to a pilot of an aircraft positional guidance in the space with reference to the ground references. Navigational aids are classified into three groups. They are

1. Long range aids

2. Short range aids

3. Terminal aids (Comparisation)

Most navigational aids concentrate on Range that is either in nmi or Km. For range measurements in space it will be in nmi. At ground based applications this will be expressed in $\mathrm{Km}$.

\section{Long range nav-aids}

LORAN C, DECCA come under this category of long range navigational aids. DECCA is a oldest electromagnetic radio position timing system. It is widely adopted in position fixing system and it takes observations from 6 transmission stations using phase differencing techniques. It is operated up to a range of $240 \mathrm{nmi}$ with accuracy of 50 to $100 \mathrm{~m}$. OMEGA is a very-long-range; very-low-frequency (VLF) radio navigation system operating in the internationally allocated navigation band between $10-14 \mathrm{KHz}$.

\section{Short range nav-aids}

The short range nav-aids are called enroute nav-aids. It defines the airways and is used for locating the reporting points. Very high frequency Omni range (VOR) is the work horse of enroute air navigation. It provides azimuthal guidance to the aircraft up to $200 \mathrm{nmi}$ and operates in the range of 108 to $118 \mathrm{MHz}$.

Terminal aids

The Terminal aids are the most sensitive aids, which helps the pilot in final phase of the landing. The guidance provided must be a very high integrity to ensure a very high probability of success for each landing. These facilities play a vital role when the visibility is poor and cloud ceiling is low. Instrument landing system (ILS) comes in this category. However, VOR is a fousing topic, its ancillary information is as follows.

\section{A.2. Applications of VOR}

The main purpose of the VOR is to provide the Navigational signals for an Aircraft receiver which will allow the pilot to determine the bearing of the Aircraft to a VOR facility. In addition to this VOR enables the air traffic controllers in the area control radar (ARSR) and (ASR) for identifying the aircraft in their scopes easily. They can monitor whether aircrafts are following the radials correctly. VOR located outside the airfield on the extended centre line of the runway would be useful for the aircraft for making a straight VOR approach. With the help of the auto pilot aircraft can be guided to approach the airport for landing. VOR located en route would be useful for air traffic to maintain their pre determined routes (PDRS) and are also used as reporting points [2] [3]. 


\section{International Journal of Innovative Research in Electrical, Electronics, Instrumentation and Control Engineering \\ ISO 3297:2007 Certified}

Vol. 5, Issue 6, June 2017

VORs located at radial distance of about 40 miles in different directions around an international airports can be used as holding VORs for regulating the aircrafts for their landing in quickest time. They would be of immense help to the aircraft for holding overhead and also to the ATCO for handling the traffic conveniently.

\section{A.3. Distinctive features of VOR}

It provides an infinite number of radial course but practically it can be said that VOR transmits a separate radial for each degree of the azimuth i.e. 360 radials. Selection of the radial of the VOR to be flown is done by the pilot himself. VOR furnishes definite course guidance TO or FROM the ground station at any

point within its service area Indication for "ON-COURSE" navigation is visual on course deviation indicator rather than aural as in the case of LF/MF radio range. The information about the radial is absolute i.e., VOR signals are independent of the altitude and heading of the aircraft.

With 59 channels available for channel spacing of $100 \mathrm{KHz}$ VORs on the same or adjacent channels can be widely separated geographically to minimize interference. $50 \mathrm{KHz}$ channel spacing is permitted for a radio frequency carrier having a tolerance of $+0.002 \%$.

It provides instantaneous bearing information to any number of aircrafts without getting saturated like DME. It offers advantages of VHF propagation i.e., it does not suffer from static or atmospheric disturbances. Hence signal to noise ratio is very good. Due VHF operation, less expensive and smaller antenna system can be used than LF/MF radio ranges.

The RF energy radiated from the VOR is horizontally polarized thus making it more immune from manmade noise as that generated by automobile ignition and electrical equipments.

\section{A.4 Equations referred for computing results}

The following are the governing equations for computing Kalman gain $\left(\mathrm{K}_{\mathrm{n}}\right)$, Expected value $\left(\mathrm{X}_{\mathrm{n}}\right)$ and Error in Expected value $\left(\mathrm{P}_{\mathrm{n}}\right)$ :

where,

$$
\begin{aligned}
& \mathrm{K}_{\mathrm{n}}=\mathrm{P}_{\mathrm{n}-1} /\left(\mathrm{P}_{\mathrm{n}-1}+\mathrm{r}\right) \\
& \mathrm{X}_{\mathrm{n}}=\mathrm{X}_{\mathrm{n}-1}+\mathrm{K}_{\mathrm{n}}\left(\mathrm{Z}_{\mathrm{n}}-\mathrm{X}\right. \\
& \mathrm{P}_{\mathrm{n}}=\left(1-\mathrm{K}_{\mathrm{n}}\right) \mathrm{P}_{\mathrm{n}-1}
\end{aligned}
$$

' $r$ ' is measurement error, ' $n$ ' is present state and ' $n-1$ ' $i$ one time step before present state.

\section{REFERENCES}

[1] Kayton M. And Fried, W., “Avionics Navigation Systems”, John Wiley \& Sons Inc., New York, $2^{\text {nd }}$ edition, 1997.

[2] ICAO PANS OPS, "Procedures for Air Navigation Services (PANS), Aircraft Operations (OPS)", Vol. 1, Flight Procedures, 1993.

[3] ICAO Annex.10, "Communication Procedures Including Those with PAN Status, Radio Navigation aids", Volume II, 1995, Amendment 89, July 2006.

[4] Peter C Sandretto, "Electronic Avigation Engineering", International Telephone and Telegraph Corporation, New York, 1958

[5] Leonid Shifrin, "Mathematica Programming: An Advanced Introduction", Version 1.01, California, 2008.

[6] Eugene Don, "Mathematica", McGraw Hill, Schaum's Outline series, $2^{\text {nd }}$ edition, 2009

[7] Wolfram Mathematica Tutorial Collection, "Visualization and Graphics", Wolfram Research Inc. 2008

[8] Wolfram Mathematica Tutorial Collection, "Core Language", Wolfram Research Inc. 2008 